\title{
On the role of verbalization during task set selection: Switching or serial order control?
}

\author{
RICHARD L. BRYCK and ULRICH MAYR \\ University of Oregon, Eugene, Oregon
}

\begin{abstract}
Recent task-switching work in which paper-and-pencil administered single-task lists were compared with task-alternation lists has demonstrated large increases in task-switch costs with concurrent articulatory suppression (AS), implicating a crucial role for verbalization during switching (Baddeley, Chincotta, \& Adlam, 2001; Emerson \& Miyake, 2003). Experiment 1 replicated this result, using computerized assessment, albeit with much smaller effect sizes than in the original reports. In Experiment 2, AS interference was reduced when a sequential cue (spatial location) that indicated the current position in the sequence of task alternations was given. Finally, in Experiment 3, switch trials and no-switch trials were compared within a block of alternating runs of two tasks. Again, AS interference was obtained mainly when the endogenous sequencing demand was high, and it was comparable for no-switch and switch trials. These results suggest that verbalization may be critical for endogenous maintenance and updating of a sequential plan, rather than exclusively for the actual switching process.
\end{abstract}

It has long been thought that language and executive regulation of thought and action are intricately linked. The Russian psychologists Lev Vygotsky and Alexander Luria (1994) proposed a relationship between the two as follows:

Thanks to the planning function of speech, geared to the child's activity, the child creates, parallel to the stimuli of his environment, a second series of auxiliary stimuli standing between him and his environment and directing his behavior. And it is due to this very secondary series of stimuli, created with the aid of speech, that the behavior of the child reaches a higher level, acquiring a relative freedom from the situation that directly attracts it, and impulsive attempts are transformed into a planned, organized behavior. (p. 111)

While certainly intriguing, research that provides a direct test of this rather broadly conceived language-control relationship is scarce. During recent years, however, empirical studies have begun to look at the role of verbal processing in executive control in the context of the socalled task-switching paradigm (Baddeley, Chincotta, \& Adlam, 2001; Emerson \& Miyake, 2003; Saeki \& Saito, 2004a). Generally, this paradigm involves a comparison between trials, or blocks of trials, in which tasks need to be switched with trials, or blocks of trials, in which tasks repeat (see, e.g., Jersild, 1927; Spector \& Biederman,

We thank Christina Ryder, Colin Maxwell, and Jennifer DeCosta for their help in running the participants in the experiments and Michael Anderson, Edward Awh, Jeffery Cottrell, and Keith Gordon for helpful comments. Correspondence concerning this article should be sent to U. Mayr, Department of Psychology, 1227 University of Oregon, Eugene, OR 97403-1227 (e-mail: mayr@darkwing.uoregon.edu).
1976). The difference between switch and no-switch trials has been termed the switch cost. The observed costs can be substantial in size and are often thought to reflect the additional executive control demands needed to reconfigure the cognitive system to currently relevant task demands (e.g., Gehring, Bryck, Jonides, Albin, \& Badre, 2003; Mayr \& Kliegl, 2000; Meiran, 1996; Rubinstein, Meyer, \& Evans, 2001).

To test the role of speech in executive control, investigators have used an articulatory suppression (AS) task, requiring the continued repetition of a familiar word or sequence (e.g., the), concurrent with performance in a task-switching paradigm. AS is thought to impair articulatory processing selectively, while leaving executive control processes proper relatively unaffected (Baddeley, Lewis, \& Vallar, 1984). Thus, a finding of selective AS interference on switch costs would suggest a role for an important subcomponent of verbal processing, articulation, during task switching.

Baddeley et al. (2001) were the first to investigate AS effects on switching, using paper-and-pencil administered lists in which participants either had to alternate between simple addition and subtraction problems within a list or had to alternate between pure addition and pure subtraction lists. A highly reliable, negative effect of a simple AS task (repetition of the) was found with the alternation lists, but only when the stimuli were bivalentthat is, when they did not contain the corresponding operator signs (i.e., + or - ) and were, therefore, perceptually ambiguous as to which task was relevant on any given trial. It is generally thought that endogenous control during task switching becomes relevant only in such ambiguous situations (Mayr, 2001; Rogers \& Monsell, 1995). 
This result is therefore consistent with the idea that articulation may be critical during endogenous control. Accordingly, the elevated switch costs during AS conditions (hereafter referred to as the $A S$ effect) can be taken to suggest that verbalization plays a critical role in the switching process.

A similar study by Emerson and Miyake (2003) used the same list paradigm and successfully replicated Baddeley et al.'s (2001) finding of increased switch costs under AS. Elevated switch costs were seen under AS, even when compared with a secondary-task control condition (not thought to interfere with normal verbal processing) in which participants were required to tap their foot while performing the arithmetic problems. Generally, similar results were reported by Saeki and Saito (2004a), again using a list paradigm.

Although these results suggest a role for verbal processing during executive task management, they do not provide a precise characterization of its functional significance. Specifically, the blocked comparison between pure alternating and pure nonalternating lists of trials confounds two aspects of task selection costs. It has been repeatedly noted that in addition to the costs that emerge at transition points between tasks (local switch costs), the mere fact of having to deal with two different tasks within the same context produces costs that are apparent even on no-switch trials (global switch costs or mixing costs; see, e.g., Fagot, 1995; Kray \& Lindenberger, 2000; Mayr, 2001). This has led some authors to draw a conceptual distinction between a task/goal decision stage, required on all trials, and the process of configuring the cognitive system when a change in task becomes necessary, with only the latter being required on switch trials (e.g., Fagot, 1995; Rubinstein et al., 2001).

In principle, verbalization may be involved in either of the two components. For example, some authors have argued that a large component of local switch costs simply reflects the long-term memory retrieval of task rules (e.g., Mayr \& Kliegl, 2000, 2003) and that this process may rely on verbal self-cuing (Emerson \& Miyake, 2003). In contrast, the global cost could reflect the working memory demands imposed by having to maintain and decide between two competing tasks. Thus, the observed AS effect on switch costs may simply reflect increased demands on a general working memory resource during task switching (but see Mayr, 2001). A more interesting possibility is suggested by a close examination of the task-switching paradigm used by Baddeley et al. (2001) and Emerson and Miyake (2003; see also Saeki \& Saito, 2004a). In the case of alternating lists and bivalent stimuli, participants switch between tasks in a manner that requires on-line control of the relevant serial order of tasks. Language, which is inherently sequential in nature, may fulfill this rather specific serial order control function. In fact, this hypothesis seems to be highly consistent with how both Baddeley et al. (2001) and Emerson and Miyake (2003) interpreted their results for AS effects on switch costs.
Our goal in the present series of experiments was to assess the role of articulation during task-switching situations and, in particular, to test the idea that the critical function might be the endogenous sequencing of tasks. However, we began in Experiment 1, with an attempt to replicate the basic AS effect on switch costs by using a refined version of the blocked switching paradigm that eliminates certain problems present in the paradigm used by Baddeley et al. (2001) and Emerson and Miyake (2003).

\section{EXPERIMENT 1}

One noteworthy aspect of the AS effect on switch costs reported by Baddeley et al. (2001) and Emerson and Miyake (2003) is the size of the effect. For example, Emerson and Miyake's study showed the articulatory suppression effect on switch costs to range from a $59 \%$ to a $150 \%$ increase, as compared with switch costs in the control condition. Although it is certainly not the case that only large effects imply that a crucial process is affected by a secondary-task manipulation, in cases in which such large effects do occur, it seems tempting to interpret them in terms of a very important, perhaps even necessary, processing component.

It is possible that the large effects shown in the earlier work were related to the way switch costs were assessed. In both Baddeley et al.'s (2001) and Emerson and Miyake's (2003) studies, a paper-and-pencil assessment was used that allowed scoring of response times (RTs) only for the whole list, and not for individual trials. Errors usually elicit additional post-error processing (see,e.g., Gehring, Goss, Coles, Meyer, \& Donchin, 1993), and this should be particularly true in lists of alternating trials where each error may require the participants to reposition themselves appropriately within the task sequence. In other words, the existing data allow no firm conclusions regarding the temporal aspects of the AS effect; in principle, the observed RT effect may be driven completely by an increase in errors within alternation blocks. Such errors, in turn, may very well reflect failures in maintaining the adequate task sequence.

We therefore attempted to replicate the AS effect on switch costs, using a computerized presentation mode that allowed for trial-by-trial measurement of RTs and errors. To adapt the tasks used by Baddeley et al. (2001) and Emerson and Miyake (2003) to a computerized procedure, we used a verification task in which participants made yes/no judgments, instead of the production method of handwriting an equation's answer. Despite the aforementioned limitations, we retained the blocked comparison between switch and no-switch conditions from the previous studies (Baddeley et al., 2001; Emerson \& Miyake, 2003), so as to not change too many aspects in a single step.

Aside from these changes, the design was very similar to that in Emerson and Miyake (2003). We used an AS task in which participants repeated the word the in time with a metronome, in an attempt to interfere with normal 
articulatory processing in working memory. We also used another secondary task, foot tapping, intended to control for potential, unspecific dual-task costs. In addition, there was a no secondary-task control condition. Finally, we also compared lists of bivalent stimuli (i.e., stimuli with no operators) with lists of univalent stimuli (i.e., stimuli with operators), in order to separate AS effects on endogenously driven processes from effects on exogenously driven processes.

\section{Method}

Participants. Experiment 1 included 24 participants, who were compensated with course credit or monetary payment (\$7) for participating in the study.

Tasks and Stimuli. The participants were required to make verification judgments to simple arithmetic equations presented within a central frame on the screen. Each equation contained a singledigit number (2-9), to which a single digit (either 1 or 2) could be either added or subtracted. The participants were instructed to make a speeded keyboard response ("/" for correct or " $z$ " for incorrect) to a probe number indicating the potential answer to the problem. Fifty percent of the probes were correct (e.g., $5-1=4$ ), and the other $50 \%$ were incorrect (e.g., $5-1=6$ ). Half of the incorrect probes were the correct answer for the opposite task (e.g., $5-2=$ 7); the other half of incorrect probes were the correct answer for the opposite modifier (e.g., $5-1=3$ ). The response-to-stimulus interval (RSI) was set to $50 \mathrm{msec}$ for half of the participants and to $250 \mathrm{msec}$ for the other half of the participants so that there were 12 participants in each RSI condition. ${ }^{1}$

The participants performed the arithmetic problems under three different conditions. In the $A S$ condition, the participants were required to repeat the word the once per beat of a metronome set to 120 beats per minute. The foot-tapping condition was designed as a dual-task control to the AS condition, in that it provided an approximately equal level of dual-task interference, yet was not thought to interfere with phonological processing (Emerson \& Miyake, 2003). In this condition, the participants were required to tap their dominant foot on a metal pan in time with the metronome (set to the same pace as in the AS condition). The third condition was the nosecondary-task control condition. Here, the metronome beat was present but was irrelevant for the task. An experimenter was present at all times to monitor both the accuracy and the timing of the participants' secondary-task performance. Experimenters also attempted to monitor the participants' response rates so that they did not synchronize their keypresses with the timing of the metronome.

For half of the blocks, the appropriate operator (plus or minus sign) was displayed on the screen (i.e., univalent stimuli). For the other half of the blocks, the operators were absent from the display (i.e., bivalent stimuli). Like previous studies, the switch variable was manipulated in a blocked manner. In other words, half of the blocks were no-switch ( pure) situations in which all the arithmetic problems were either addition or subtraction (with equal numbers of each presented throughout the experiment for each participant); the other half of the blocks were switch (mixed) situations in which the participants were required to alternate between addition and subtraction problems throughout the block.

The stimuli were displayed on $800 \times 600$ resolution Macintosh computers, using PsyScript software (Bates \& D'Oliveiro, 2003). Each digit in the stimulus display was about $9 \mathrm{~mm}$ high $\times 6 \mathrm{~mm}$ wide. The stimuli appeared at a viewing distance of about $60 \mathrm{~cm}$.

Procedure. Twelve unique block types were constructed by crossing the three secondary-task variables (AS, foot tapping, or control) with the two types of stimulus ambiguity (univalent or bivalent) and the two switch conditions (no switch or switch); each block contained 12 trials. These 12 block types were cycled through six times within the experiment. Within each cycle, no-switch and switch blocks for a given stimulus ambiguity $\times$ secondary-task constellation were always presented in back-to-back pairs. Across the six cycles, the order in which switch and no-switch trials were presented was counterbalanced. Within each switch/no-switch pair, the no-switch block required either addition only or subtraction only. However, whether addition or subtraction served as the noswitch task for a particular stimulus ambiguity $\times$ secondary-task constellation was balanced across cycles, within each participant. In total, there were 864 total trials in this experiment (12 block types $\times 12$ trials each $\times 6$ cycles).

The stimuli were presented within a single, centrally located box. For switch blocks, the order of tasks remained constant throughout the experiment, always starting with addition and alternating operations on each trial (i.e., addition, subtraction, addition, subtraction, ....).

The experiment began with instructions regarding the primary tasks and then with 24 practice trials of bivalent stimuli (with no secondary task); half of the trials were in a no-switch block, and the other half were in a switch block. The participants received further practice during the first iteration of the experiment by performing a short practice block ( 6 trials each) before each of the 12 block types. In addition to providing practice with the secondary tasks, these practice blocks familiarized the participants with each of the 12 block types and the differential demands of univalent versus bivalent stimuli. The participants were also given the chance to practice each of the secondary tasks alone, in time with the metronome, before each short practice block. This allowed them to familiarize themselves with the metronome beat, without the added dual-task difficulty of the arithmetic problems.

In the event of an inaccurate response, auditory feedback was presented. In the case of bivalent stimuli blocks, the correct task cue $(+$ or -$)$ was also shown. After a minimum delay of $1 \mathrm{sec}$ and a keypress, the block continued. The participants were instructed to prepare for the next trial in the sequence if they made an error.

\section{Results}

Mean RTs and mean percentage of errors were calculated for each of the 12 critical conditions across participants. The initial practice block and the subsequent 12 short practice blocks were excluded from all the analyses. Trimmed means were calculated by excluding any trials that were 2.5 standard deviations above or below each participant's mean in each of the 12 conditions. This trimming resulted in the loss of $2.9 \%$ of the data. The first trial in a block was also taken out of the analysis, because it was neither a true no-switch nor a switch trial, as were error trials and trials after errors.

To analyze the data, we used an analysis of variance (ANOVA) that included the switch variable (switch vs. no switch), the ambiguity variable (univalent vs. bivalent), and two nonorthogonal contrasts to characterize the secondary-task effects. The first contrast compared the control condition with the foot-tapping condition (foot-tapping contrast); the second, critical contrast compared foot tapping with AS (AS contrast). Splitting the three-level secondary-task variable into these two contrasts allowed targeted assessments of (1) unspecific secondary-task effects through the foot-tapping contrast and (2) the specific effects of AS, relative to foot tapping, in the AS contrast. Analyses with these two contrasts are identical with two separate $2 \times 2 \times 2$ ANOVA for all effects that involve these two contrasts, whereas all the remaining effects are tested across the three secondary-task groups contained in the overall design. 


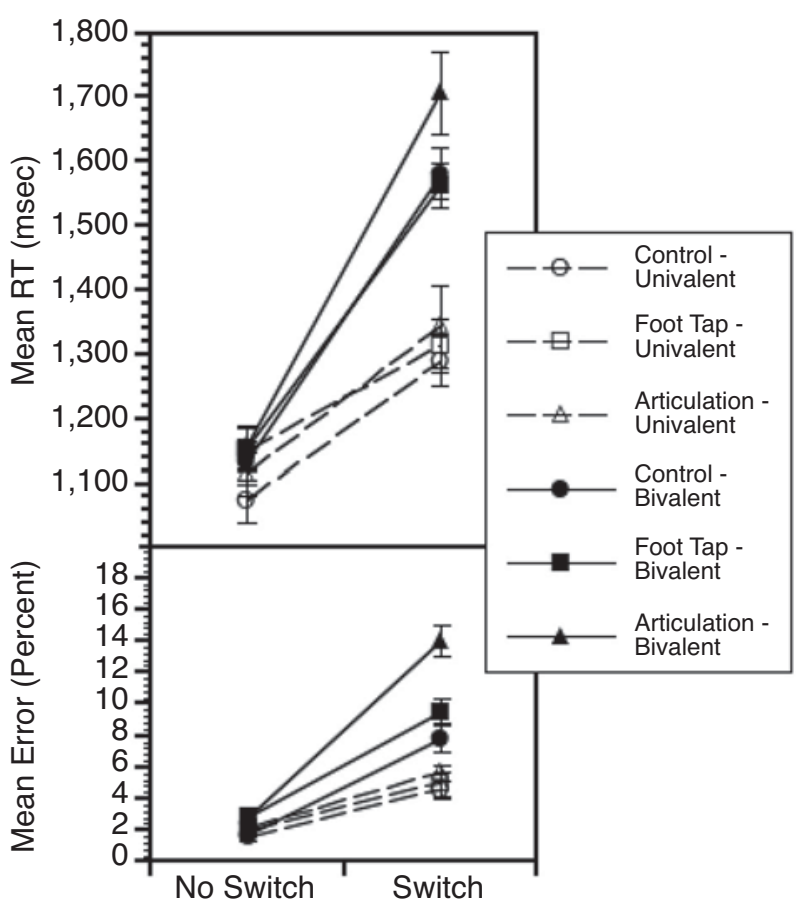

Figure 1. Mean response times (RTs, top) and percentages of error (bottom) for Experiment 1 as a function of switch/no switch, stimulus ambiguity, and secondary-task condition. Error bars reflect one standard error of the mean.

The relevant RTs and error scores are presented in Figure 1; the results of the statistical analysis are contained in Table 1. In addition, switch costs for all the experiments combined are presented in Table 2. We will focus here and throughout on the theoretically relevant effects and will discuss additional effects and lower order interactions only as they qualify the critical effects. As can be seen from Figure 1 and Table 2, we were able to replicate typical task-switching effects: We obtained substantial switch costs for both RTs and errors, and these were significantly larger for bivalent stimuli (see Table 1).
The primary question is to what degree switch costs are affected by the secondary tasks. As can be seen in both Figure 1 and Table 2, foot tapping did not lead to an increase of switch costs, either for RTs or for errors (in fact, for RTs the effect was in the opposite direction). However, for the AS condition, switch costs increased reliably for both RTs and errors, as compared with foot tapping. Critically, this effect was specific to the bivalent stimulus condition. The corresponding interaction between the AS contrast, switch, and stimulus ambiguity was reliable both for RTs and errors (see Table 1).

\section{Discussion}

The results of Experiment 1 replicate and extend the previous work on the effects of AS on task switching (Baddeley et al., 2001; Emerson \& Miyake, 2003). AS affected switch costs beyond the costs produced through a nonverbal secondary task. Furthermore, this effect was limited to situations in which endogenous control was necessary - that is, when the stimuli where bivalent. Importantly, this effect was obtained despite considerable changes to the paradigm (i.e., verification, rather than production procedure, and computerized testing).

The AS effect on switch costs was, however, considerably smaller than that found in the earlier studies (Baddeley et al., 2001; Emerson \& Miyake, 2003). For example, RT switch costs in the present experiment increased by $23 \%$ as a function of AS, whereas the corresponding values for Emerson and Miyake ranged from 59\% to $150 \%$.

One possible reason why AS effects on RTs were relatively small was raised in the review process and is related to our use of a verification task (rather than a production task, as in previous studies). For a verification task to work in a task-switching situation, it is necessary to include foils that constitute correct answers to the currently irrelevant task (e.g., $6-3=9$ ). However, such foils are known to produce a Stroop-like interference effect and, therefore, may have increased the tendency to make errors that otherwise might have shown up as longer RTs (see, e.g., Zbrodoff \& Logan, 1986). To examine this possibility, we reanalyzed the results in Experiment 1

Table 1

Analysis of Variance Table for Experiment 1

\begin{tabular}{|c|c|c|c|c|c|c|}
\hline \multirow[b]{2}{*}{ Analysis and Source of Variance } & \multicolumn{3}{|c|}{ RT } & \multicolumn{3}{|c|}{ Error } \\
\hline & $F$ & $M S_{\mathrm{e}}$ & $p$ & $F$ & $M S_{\mathrm{e}}$ & $p$ \\
\hline Ambiguity & 60.82 & 399,843 & $.000^{* *}$ & 75.62 & 102 & $.000^{* *}$ \\
\hline Control vs. foot tapping (C1) & 1.36 & 200,530 & .256 & 5.35 & 56 & $.030^{*}$ \\
\hline Foot tapping vs. suppression (C2) & 1.80 & 259,065 & .193 & 8.42 & 86 & $.008^{* *}$ \\
\hline Switch & 368.64 & 265,761 & $.000^{* *}$ & 166.26 & 159 & $.000^{* *}$ \\
\hline Ambiguity $\times \mathrm{C} 1$ & 4.45 & 54,670 & $.046^{*}$ & 1.82 & 52 & .190 \\
\hline Ambiguity $\times \mathrm{C} 2$ & 11.82 & 48,770 & $.002^{* *}$ & 7.24 & 44 & $.013^{*}$ \\
\hline Ambiguity $\times$ switch & 75.52 & 204,749 & $.000^{* *}$ & 55.73 & 90 & $.000^{* *}$ \\
\hline Switch $\times \mathrm{C} 1$ & 4.96 & 44,001 & $.036^{*}$ & 0.06 & 75 & .802 \\
\hline Switch $\times \mathrm{C} 2$ & 19.67 & 53,711 & $.000^{* *}$ & 13.48 & 43 & $.001^{* *}$ \\
\hline Ambiguity $\times$ switch $\times \mathrm{C} 1$ & 0.19 & 27,058 & .667 & 0.21 & 60 & .651 \\
\hline Ambiguity $\times$ switch $\times \mathrm{C} 2$ & 4.49 & 31,096 & $.045^{*}$ & 5.79 & 63 & $.025^{*}$ \\
\hline
\end{tabular}

Note- $\mathrm{C} 1$ = Contrast 1 (foot-tapping contrast), which compared control (no secondary task) and foot tapping; $\mathrm{C} 2=$ Contrast 2 (AS contrast), which compared foot-tapping and articulatory suppression secondary-task conditions. Degrees of freedom $(d f)$ for all conditions were $1,23 .{ }^{*} p<.05 .{ }^{* *} p<.01$. 
Table 2

Summary of Descriptive Statistics for Switch Costs for Each Experiment and Secondary-Task Condition

\begin{tabular}{|c|c|c|c|c|c|c|c|c|c|c|c|c|}
\hline \multirow[b]{3}{*}{ Condition } & \multicolumn{4}{|c|}{ Control } & \multicolumn{4}{|c|}{ Foot Tapping } & \multicolumn{4}{|c|}{ Articulatory Suppression } \\
\hline & \multicolumn{2}{|c|}{$\begin{array}{l}\text { RT Cost } \\
(\mathrm{msec})\end{array}$} & \multicolumn{2}{|c|}{$\begin{array}{c}\text { Error Cost } \\
(\%)\end{array}$} & \multicolumn{2}{|c|}{$\begin{array}{c}\text { RT Cost } \\
(\mathrm{msec})\end{array}$} & \multicolumn{2}{|c|}{$\begin{array}{c}\text { Error Cost } \\
(\%)\end{array}$} & \multicolumn{2}{|c|}{$\begin{array}{l}\text { RT Cost } \\
(\mathrm{msec})\end{array}$} & \multicolumn{2}{|c|}{$\begin{array}{c}\text { Error Cost } \\
(\%)\end{array}$} \\
\hline & $M$ & $S D$ & $M$ & $S D$ & $M$ & $S D$ & $M$ & $S D$ & $M$ & $S D$ & $M$ & $S D$ \\
\hline \multicolumn{13}{|c|}{ Experiment 1: Local Switch Costs } \\
\hline Uni & 217 & 67 & 3.05 & 2.54 & 162 & 88 & 2.91 & 3.74 & 229 & 121 & 3.43 & 3.60 \\
\hline $\mathrm{Bi}$ & 450 & 176 & 6.08 & 5.20 & 409 & 159 & 6.67 & 4.05 & 552 & 232 & 11.09 & 5.25 \\
\hline \multicolumn{13}{|c|}{ Experiment 2: Local Switch Costs } \\
\hline Low Seq & 368 & 181 & 3.20 & 3.46 & 435 & 246 & 7.08 & 5.92 & 514 & 228 & 7.13 & 4.41 \\
\hline High Seq & 460 & 184 & 5.54 & 5.22 & 531 & 243 & 10.05 & 4.85 & 579 & 224 & 16.13 & 8.29 \\
\hline \multicolumn{13}{|c|}{ Experiment 3: Local Switch Costs } \\
\hline Low Seq, Uni & 84 & 98 & 0.72 & 2.86 & 108 & 130 & 1.25 & 4.32 & 57 & 136 & 2.12 & 5.49 \\
\hline Low Seq, Bi & 151 & 148 & 2.34 & 6.31 & 168 & 125 & 4.58 & 4.58 & 225 & 193 & 2.98 & 3.55 \\
\hline High Seq, Uni & 85 & 114 & 1.35 & 3.29 & 73 & 111 & 1.77 & 3.48 & 107 & 145 & 1.27 & 4.90 \\
\hline High Seq, Bi & 216 & 146 & 1.07 & 3.12 & 243 & 170 & 2.25 & 4.90 & 264 & 231 & 2.49 & 4.69 \\
\hline \multicolumn{13}{|c|}{ Experiment 3: Global Switch Costs } \\
\hline Low Seq & 146 & 128 & 1.89 & 3.34 & 176 & 136 & 2.36 & 2.51 & 189 & 154 & 1.56 & 2.42 \\
\hline High Seq & 182 & 169 & 2.60 & 3.60 & 179 & 156 & 3.85 & 4.08 & 173 & 184 & 5.72 & 4.74 \\
\hline
\end{tabular}

Note-Uni, univalent stimuli; Bi, bivalent stimuli; Low Seq, low sequencing load; High Seq, high sequencing load; local switch costs, difference between switch and no-switch conditions; global switch costs, difference between bivalent and univalent conditions (collapsed across no-switch and switch conditions).

after eliminating trials with the Stroop-like foils. Switch costs were generally smaller in this situation (by about $50 \mathrm{msec}$ ), but the AS effect on switch costs was almost identical whether or not the Stroop-like foils were included (without foils, $113 \mathrm{msec}$; with foils, $102 \mathrm{msec}$ ). (We repeated this analysis for Experiments 2 and 3, with very similar results).

As was mentioned in the introduction to this experiment, another possible reason for the difference in switch costs across experiments is that post-error recovery processes, which may have inflated switch cost estimates in the blocked switching paradigm, do not affect switch costs in the computerized paradigm. Unfortunately, we cannot directly test this hypothesis, because we did not record the time the participants spent processing the visual realignment aid we provided in case of an error. However, in principle, it is an interesting and easily testable idea that AS interferes specifically with participants' attempts to reestablish the adequate sequential plan after errors.

Finally, a third possibility is that the difference between pure lists of arithmetic production (e.g., addition only) and an alternation situation may constitute a particularly strong contrast between highly automatized processing, on the one hand, and controlled processing, on the other. After all, simple arithmetic production is extremely overlearned, and for that very reason, the constant interruption of automatic processing, as required in the alternation condition, may be particularly hard. In contrast, for the verification task, even pure-list situations are less automatic, because they contain an arbitrary stimulusresponse component (e.g., pressing one key for correct, the other for incorrect problems). Note that even purelist RTs were relatively long. Thus, in comparison with an arithmetic production task, the addition of active, topdown sequencing processes may be lower when going from pure list to alternation, simply because, to some degree, these processes are already required for the pure verification lists.

Irrespective of the question of the size of the AS effect, the fact that we were able to establish the basic AS effect on switch costs within our paradigm reinforces the hypothesis of a relationship between language processes and executive control. The aim of the next experiment was to provide a more detailed characterization of this relationship.

\section{EXPERIMENT 2}

In Experiment 2, we attempted a direct test of the hypothesis that verbal processing serves a sequencing function during task switching. We used the blocked switching paradigm, as in Experiment 1; in addition, we manipulated the sequencing demand through spatial sequence cues.

In the low-sequencing condition, two boxes of equal area were presented side by side. Starting in the left box, stimuli alternated between each box on every trial. Thus, during switch blocks, each task appeared in a unique location, allowing for exogenous cuing of the relevant task (i.e., addition was always on the left). Although the participants still had to switch between task rules endogenously in this situation (assuming bivalent stimuli), internal sequencing of tasks was not necessary. It is important to note that similar kinds of external sequencing aids have been used routinely during task-switching studies, in order not to confound switching demands with the demands of maintaining an adequate task sequence (see, e.g., Mayr \& Kliegl, 2000; Rogers \& Monsell, 1995). 
We contrasted this condition with a high-sequencing condition, which was basically identical to the situation in Experiment 1, that contained only one centrally presented box for stimulus presentation. Here, no external information regarding the task order was provided, so that the adequate sequence had to be maintained and updated internally. The only other change in design for Experiment 2 was the removal of the stimulus ambiguity variable, in order to accommodate sequencing demand as a within-subjects variable. Thus, only bivalent stimuli were used here.

\section{Method}

Participants and Design. The participants were 24 students recruited from the University of Oregon community. Compensation for participation in the study was either course credit or \$7.

Tasks and Stimuli. The same tasks and stimuli were used as in Experiment 1 . However, only bivalent stimuli were presented, and the sequence load was manipulated in a within-subjects manner. This was done by presenting stimuli that appeared in one central box only (high sequencing load) or stimuli that alternated between two side-by-side boxes (low sequencing load).

Procedure. The procedure for Experiment 2 was identical to that in Experiment 1, except that the stimulus ambiguity variable was replaced by the sequence load variable. For each participant, high or low sequencing load blocks were presented during either the first or the second half of the experiment (the order was counterbalanced evenly across participants). Within these two halves of the experiment, six different counterbalancing sequences (i.e., 3 secondarytask conditions $\times 2$ switch/no-switch blocks) iterated through six cycles (three in each half). With a block length of 24 trials, this again resulted in a total of 864 trials. No-switch blocks again alternated between pure addition and pure subtraction problems, with the order counterbalanced across the experiment for each participant. The same practice regime was used as in Experiment 1, except that it was now split between each half of the experiment (i.e., practice was done before the low-sequencing half and before the highsequencing half).

\section{Results}

The results from Experiment 2 were analyzed in the same manner as were those in Experiment 1, with the sequence load variable replacing the stimulus ambiguity variable. Data exclusion, through the same trimming procedure as that used in Experiment 1, resulted in the loss of $3 \%$ of the data. Again, error trials and trials after errors were excluded from the analysis of RT data.

As is shown in Figure 2 and Table 2, we again observed large switch costs, in both the RT and the error data. Switch costs were also generally larger for the high

\section{Low sequencing load High sequencing load}

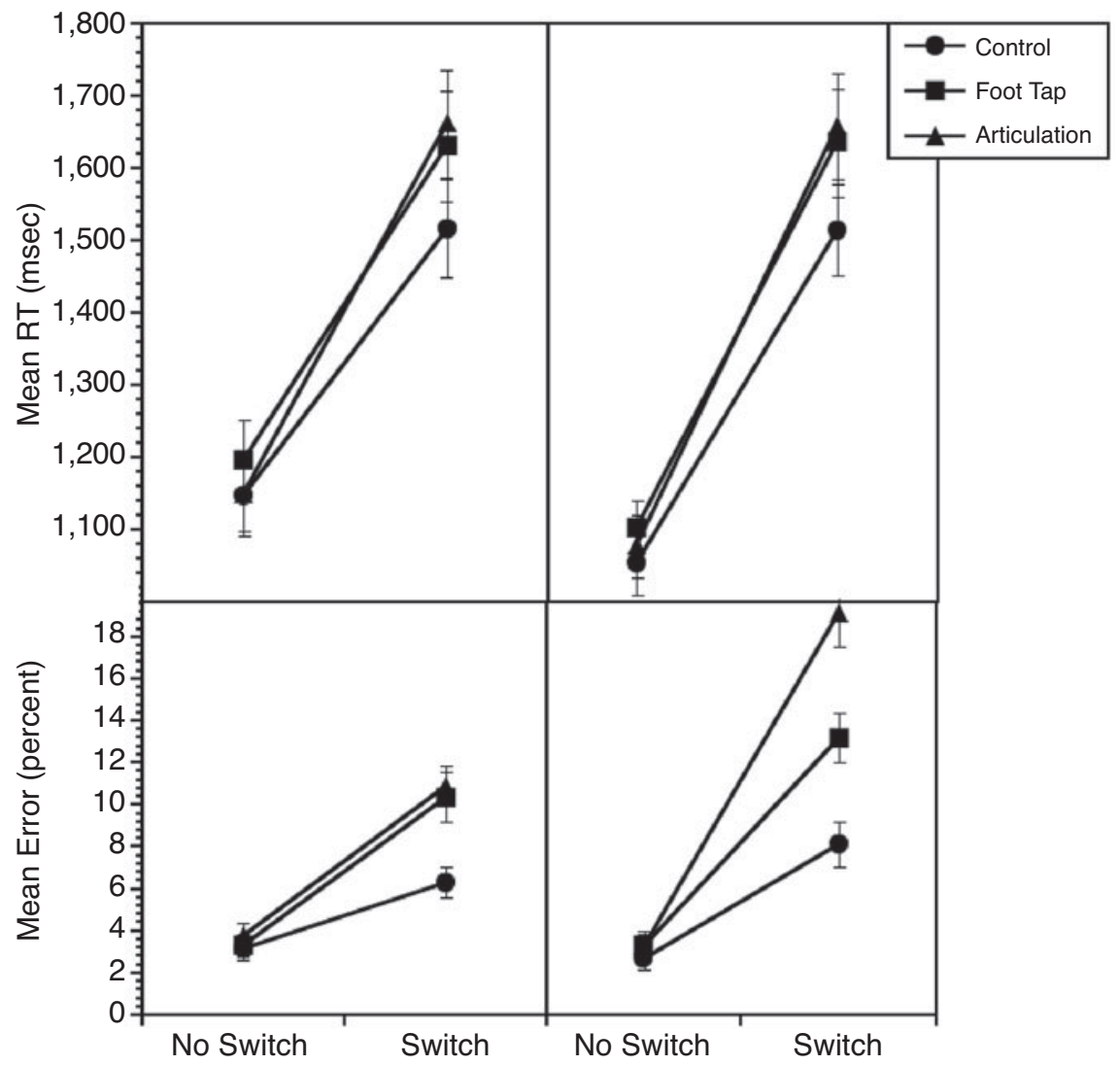

Figure 2. Mean response times (RT, top) and percentages of error (bottom) for Experiment 2 as a function of sequence load, switch/no switch, stimulus ambiguity, and secondarytask condition. Error bars reflect one standard error of the mean. 
Table 3

Analysis of Variance Table for Experiment 2

\begin{tabular}{|c|c|c|c|c|c|c|}
\hline \multirow[b]{2}{*}{ Analysis and Source of Variance } & \multicolumn{3}{|c|}{ RT } & \multicolumn{3}{|c|}{ Error } \\
\hline & $F$ & $M S_{\mathrm{e}}$ & $p$ & $F$ & $M S_{\mathrm{e}}$ & $p$ \\
\hline Sequence & 0.93 & $1,744,844$ & .345 & 8.84 & 446 & $.007^{* *}$ \\
\hline Control vs. foot tapping (C1) & 11.29 & 233,037 & $.003^{* *}$ & 22.40 & 106 & $.000^{* *}$ \\
\hline Foot tapping vs. suppression (C2) & 0.04 & 185,711 & .836 & 10.06 & 119 & $.004^{* *}$ \\
\hline Switch & 272.53 & 734,087 & $.000^{* *}$ & 183.99 & 315 & $.000^{* *}$ \\
\hline Sequence $\times \mathrm{C} 1$ & 0.01 & 108,949 & .905 & 0.54 & 107 & .468 \\
\hline Sequence $\times \mathrm{C} 2$ & 0.04 & 106,800 & .847 & 5.43 & 108 & $.029^{*}$ \\
\hline Sequence $\times$ switch & 3.51 & 442,496 & .074 & 21.68 & 227 & $.000^{* *}$ \\
\hline Switch $\times \mathrm{C} 1$ & 5.46 & 83,993 & $.029^{*}$ & 22.35 & 76 & $.000^{* *}$ \\
\hline Switch $\times \mathrm{C} 2$ & 4.15 & 93,053 & .053 & 8.64 & 104 & $.007^{* *}$ \\
\hline Sequence $\times$ switch $\times \mathrm{C} 1$ & 0.00 & 110,009 & .946 & 0.15 & 65 & .704 \\
\hline Sequence $\times$ switch $\times \mathrm{C} 2$ & 0.21 & 109,666 & .655 & 6.83 & 128 & $.016^{*}$ \\
\hline
\end{tabular}

Note - $\mathrm{C} 1$ = Contrast 1 (foot-tapping contrast), which compared control (no secondary task) and foot tapping; $\mathrm{C} 2=$ Contrast 2 (AS contrast), which compared foot-tapping and articulatory suppression secondary-task conditions. Degrees of freedom $(d f)$ for all conditions were $1,23 .{ }^{*} p<.05 .{ }^{* *} p<.01$.

sequence load condition than for the low sequence load condition (see Table 2), an effect that did not meet the reliability criterion for RTs but that was highly reliable for errors (see Table 3). As is indicated by the reliable interaction between the foot-tapping contrast $(\mathrm{C} 1)$ and the switch variable, the addition of a secondary task increased switch costs, as compared with the control condition. However, there was no reliable interaction between the sequencing load and the foot-tapping contrast (C1).

Again, the most important effects central to our hypotheses are those involving the AS contrast (C2). In terms of RTs, there was a marginally reliable interaction between the AS contrast and switching $(p=.053)$. Numerically, this effect was relatively small $(M=62 \mathrm{msec}$, $12 \%$ ), and it was not further modulated through sequencing demands. However, in terms of errors, there was a reliable interaction between the AS contrast and the switch variable that was further modulated by the sequence load variable (see Table 3). As is evident from Figure 2 and Table 2, error switch costs were particularly large in the high sequence demand condition

\section{Discussion}

The results from Experiment 2 clearly demonstrated an AS effect on accuracy when sequencing load was high. In contrast, when external spatial information cued the adequate task sequence, there was no performance difference between the AS condition and the foot-tapping condition. Thus, it appears that verbalization is critical only in situations in which the task context is not provided by the external environment. This conditional effect of AS supports our hypothesis, then, that verbal processing is critical for the maintenance/updating of task order-specifically, when other bottom-up mechanisms do not prompt the relevant task context.

At the same time, with the nearly reliable effect of AS on switch costs irrespective of sequencing demands, these data do not allow us to rule out the possibility that AS may also have more subtle effects on switch costs per se.

\section{EXPERIMENT 3}

The results from Experiment 2 support the idea that verbalization is critical in task-switching situations that require endogenous sequencing. In this context, it is important to note that the blocked switching design used so far confounds the processing demands incurred by an actual switch in task (i.e., those reflected in local switch costs) with the processing demands related to trial-to-trial endogenous selection when two or more tasks are present in the same context (i.e., reflected in global/mixing costs). Thus, the extent to which the AS specifically affects the actual switching process remains to be tested. Interestingly, on the basis of the sequencing hypothesis, there is not much a priori reason to expect a switchspecific effect. After all, participants need to know what the current task is on every trial, whether it is a switch or not.

In Experiment 3 we combined a manipulation of sequencing demands, as in Experiment 2, with the so-called alternate-runs paradigm (Rogers \& Monsell, 1995), in which runs of two same-task trials alternated (i.e., an A, A, B, B ordering of tasks). This paradigm allows a comparison between switch and no-switch trials within the same block, thus avoiding a confound between local and global costs. We also included a blocked manipulation of stimulus ambiguity. Given that blocks with univalent stimuli require little in terms of endogenous trial-to-trial selection, the contrast between univalent and bivalent stimuli (across no-switch and switch trials) allows us to assess global switch costs. In contrast to the simple comparison between single-task and mixed-task blocks, this way of assessing global costs allows a targeted assessment of endogenous selection processes, while keeping the number of relevant tasks constant (Mayr, 2001).

To summarize, with this design, we can test to what degree the sequencing demand interacts with the demands of switching between tasks, to what degree AS interacts with the sequencing demand (i.e., allowing a replication of Experiment 2), and to what degree AS, switch costs (local or global), and sequencing demands interact. 


\section{Method}

Participants. The participants were 48 University of Oregon students who were compensated with either course credit or $\$ 7$ for participating in the study.

Tasks and Stimuli. The most important change in this experiment was the use of an alternate-runs paradigm with alternating runs of two tasks, instead of a blocked comparison between switch and no-switch trials. As in Experiment 1, this experiment included a stimulus ambiguity manipulation. As in Experiment 2, this experiment included a manipulation of the sequence load - now, however, as a between-subjects variable. In the low sequencing load group, a four-quadrant square grid (two $\times$ two array of equal-sized boxes) was presented centrally, and the stimuli appeared serially in each of the quadrants in a clockwise sequence (Rogers \& Monsell, 1995). The first trial of a block always started in the upper-left quadrant for each block, so that the addition task always occurred in the top row and the subtraction task always in the bottom row. In contrast, in the high sequencing load group, the grid displayed contained only two quadrants. The stimuli thus alternated between the two quadrants, starting in the left box at the start of a block, but the overall task order remained constant (A, A, B, B). Although the spatial position was informative regarding whether a given trial was a switch or a no-switch trial (i.e., the left box vs. the right box), adequate sequencing of tasks was still necessary.
We used this procedure in order to keep it as similar as possible to the high sequencing load condition in Experiment 2, where there was no ambiguity regarding whether a given trial was a switch trial or not (because all the trials in a block were either switch or noswitch trials); however, there was still potential ambiguity regarding which task was relevant. The same three secondary-task conditions were used as in Experiments 1 and 2.

Procedure. The same practice regime was used as in Experiments 1 and 2. Also, the same counterbalancing scheme was used as in Experiment 2, except that the stimulus valance variable replaced the sequence load variable. The total trial number again was 864 . To ensure that the participants did not lose the task sequence, a display was presented above the stimulus array on error trials that indicated the current position in the sequence. This display consisted of a simple visual representation of the task sequence $(++--)$, with a red line underneath the current position.

\section{Results}

The same exclusion criteria were applied as in Experiments 1 and 2, resulting in the elimination of approximately $3 \%$ of the RTs. The relevant data for all the conditions are presented in Figure 3 and Table 4.

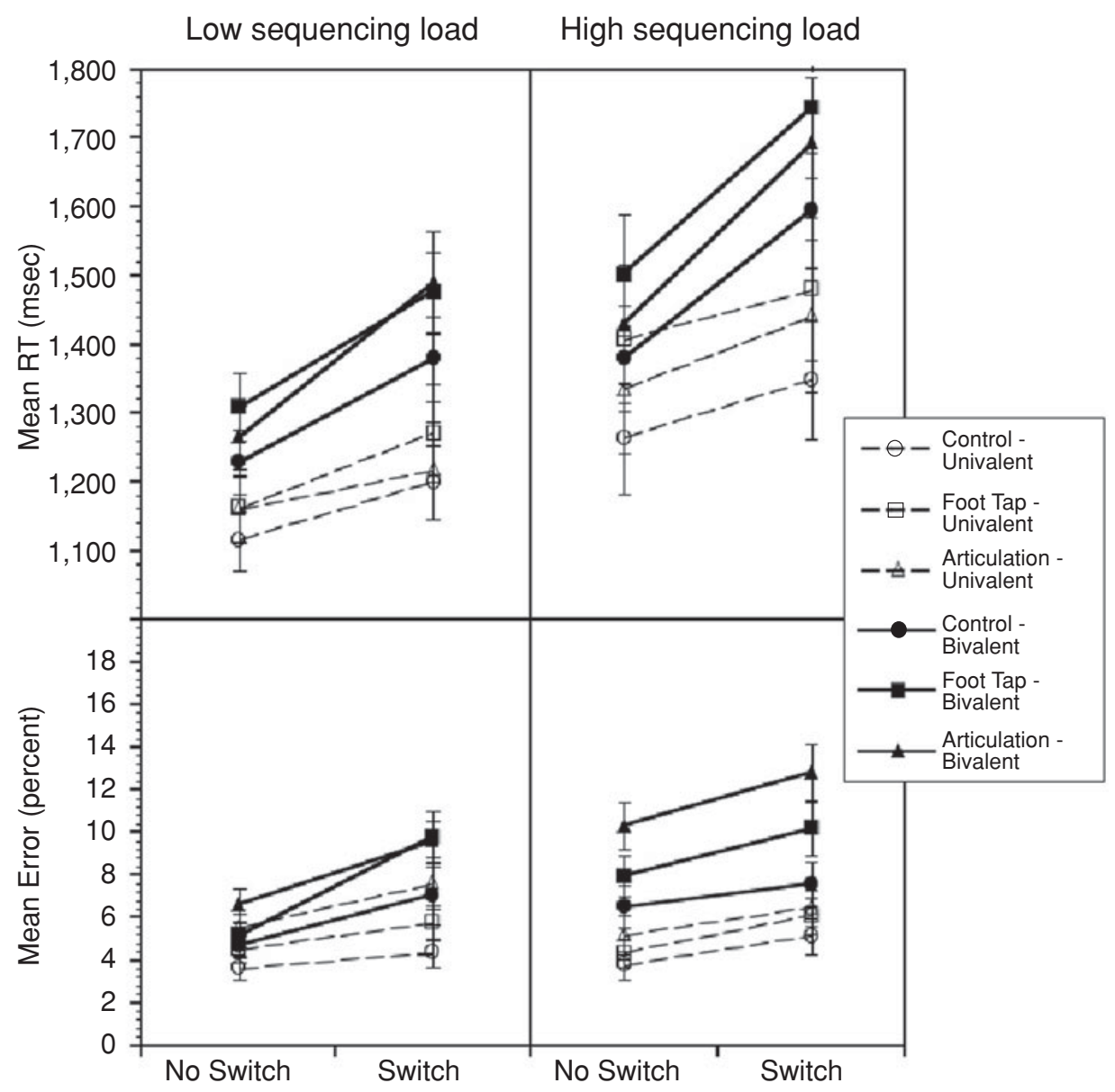

Figure 3. Mean response times (RTs, top) and percentages of error (bottom) for Experiment 3 as a function of sequence load, switch/no switch, stimulus ambiguity, and secondary-task condition. Error bars reflect one standard error of the mean. 
Table 4

Analysis of Variance Table for Experiment 3

\begin{tabular}{|c|c|c|c|c|c|c|}
\hline \multirow[b]{2}{*}{ Analysis and Source of Variance } & \multicolumn{3}{|c|}{ RT } & \multicolumn{3}{|c|}{ Error } \\
\hline & $F$ & $M S_{\mathrm{e}}$ & $p$ & $F$ & $M S_{\mathrm{e}}$ & $p$ \\
\hline Ambiguity (Amb) & 90.28 & $4,368,274$ & $.000^{* *}$ & 45.11 & 1,291 & $.000^{* *}$ \\
\hline Control vs. foot tapping (C1) & 39.71 & $1,062,183$ & $.000^{* *}$ & 26.09 & 182 & $.000^{* *}$ \\
\hline Foot tapping vs. AS (C2) & 7.68 & 155,848 & $.008^{* *}$ & 13.71 & 157 & $.001^{* *}$ \\
\hline Switch & 91.41 & $3,176,564$ & $.000^{* *}$ & 30.76 & 586 & $.000^{* *}$ \\
\hline Low vs. high sequence (Seq) & 3.67 & $17,849,830$ & .062 & 1.05 & 1,594 & .311 \\
\hline $\mathrm{Amb} \times \mathrm{C} 1$ & 0.47 & 4,415 & .494 & 3.85 & 18 & .056 \\
\hline $\mathrm{Amb} \times \mathrm{C} 2$ & 0.02 & 291 & .892 & 1.73 & 7 & .195 \\
\hline Amb $\times$ switch & 63.10 & 564,690 & $.000^{* *}$ & 5.70 & 52 & $.021^{*}$ \\
\hline Switch $\times \mathrm{C} 1$ & 1.39 & 4,551 & .245 & 3.45 & 29 & .070 \\
\hline Switch $\times \mathrm{C} 2$ & 1.38 & 5,536 & .247 & 0.18 & 1 & .675 \\
\hline Seq $\times$ Amb & 0.04 & 580,646 & .839 & 5.66 & 344 & $.022^{*}$ \\
\hline Seq $\times$ switch & 1.10 & 417,020 & .299 & 0.75 & 229 & .392 \\
\hline Seq $\times C 1$ & 3.57 & 213,976 & .065 & 0.00 & 56 & .953 \\
\hline $\mathrm{Seq} \times \mathrm{C} 2$ & 1.58 & 162,315 & .215 & 0.52 & 92 & .474 \\
\hline $\mathrm{Amb} \times \mathrm{switch} \times \mathrm{C} 1$ & 0.23 & 1,335 & .633 & 1.50 & 9 & .227 \\
\hline $\mathrm{Amb} \times$ switch $\times \mathrm{C} 2$ & 1.79 & 13,728 & .187 & 0.60 & 5 & .444 \\
\hline $\mathrm{Seq} \times \mathrm{Amb} \times \mathrm{C} 1$ & 0.65 & 74,458 & .424 & 0.78 & 37 & .381 \\
\hline $\mathrm{Seq} \times \mathrm{Amb} \times \mathrm{C} 2$ & 0.15 & 23,539 & .696 & 10.68 & 32 & $.002 * *$ \\
\hline $\mathrm{Seq} \times \mathrm{Amb} \times$ switch & 2.99 & 107,396 & .091 & 2.10 & 110 & .154 \\
\hline Seq $\times$ switch $\times$ C1 & 0.30 & 26,227 & .584 & 0.24 & 66 & .624 \\
\hline Seq $\times$ switch $\times \mathrm{C} 2$ & 0.88 & 32,197 & .354 & 0.04 & 66 & .840 \\
\hline $\mathrm{Seq} \times \mathrm{Amb} \times$ switch $\times \mathrm{C} 1$ & 0.56 & 46,133 & .457 & 0.22 & 49 & .638 \\
\hline Seq $\times$ Amb $\times$ switch $\times C 2$ & 2.86 & 61,243 & .097 & 2.05 & 60 & .159 \\
\hline
\end{tabular}

Note-C1 = Contrast 1 (foot-tapping contrast), which compared control (no secondary task) and foot tapping; $\mathrm{C} 2$ = Contrast 2 (AS contrast), which compared foot-tapping and articulatory suppression secondarytask conditions. Seq = low-sequencing group versus high-sequencing group (between-subjects factor). Degrees of freedom $(d f)$ for all conditions were $1,46 .{ }^{*} p<.05 .{ }^{* *} p<.01$.

Large effects of switching and stimulus ambiguity, as well as an interaction between these two variables, were obtained both for RTs and errors. When considering the general influence of the sequencing load variable, we found that under high sequence load, the participants responded more slowly $(M=1,467 \mathrm{msec}, S D=430)$ than under low sequence load $(M=1,272 \mathrm{msec}, S D=250)$, a difference that was marginally reliable $(p=.062)$. No comparable effect was obtained for errors. However, for errors (and not for RTs), the sequence load variable interacted with the stimulus ambiguity variable, since the difference in percentages of errors (bivalent minus univalent stimuli) was reliably larger for the high-sequence group (difference $=4.06 \%$ ) than for the low-sequence group (difference $=1.93 \%$; see Table 4 ).

Turning to the secondary-task effects, the participants generally performed more slowly and with more errors under foot tapping than under the no-secondary-task control condition in general. No additional effects involving the foot-tapping contrast $(\mathrm{C} 1)$ were reliable. Importantly, as in Experiment 2, AS affected endogenous selection in a general manner when sequencing demands were high, since there was a highly reliable three-way interaction between the AS contrast, the stimulus ambiguity variable, and the sequencing load variable for errors (see Table 4). Again, as in Experiment 2, the corresponding effect for RTs was far from reliable. For neither RTs nor errors was the corresponding effect (ambiguity $X$ AS contrast) reliable when the low sequence load group alone was analyzed.
From the foregoing analysis, it seems that AS affects only global switching costs, and not local switching costs, albeit for errors only. However, before accepting this conclusion, we need to address the additional question whether to what degree the AS effect on selection costs for the ambiguous, high sequence load condition was further modulated by the switch variable. The four-way interactions between AS contrast, stimulus ambiguity, sequencing and switching is thus the critical contrast for answering this question. Table 4 reveals effects that seem to approach significance for both RTs and errors ( $p=$ .097 and .157, respectively). However, as inspections of Figure 3 and Table 2 show, these effects reflect opposing trends for RTs and errors. Thus, although these effects might suggest a complex speed-accuracy tradeoff, they do not show a specific effect of AS on bivalent switch trials under a high sequencing load. Furthermore, even regardless of the sequencing variable, the three-way interactions between AS contrast, stimulus ambiguity, and switching for RTs and errors are far from reliable. In other words, the effect of AS on endogenous task control seemed to be fairly similar for no-switch and switch trials.

\section{Discussion}

There were several critical findings in this experiment. First, we replicated the effect of AS on errors for the situation in which endogenous sequential control was necessary (i.e., bivalent stimuli and absence of sequential cuing). This result strengthens the general idea that verbal processing contributes a specific sequencing func- 
tion to the general process of selecting an appropriate task set. As in Experiment 2, this effect was confined to errors. We do not have a good explanation for why errors seem more sensitive to AS than RTs are. It is, however, noteworthy that such a selective sensitivity of one measure over the other would have remained undetected in the earlier reports in which RTs could not be assessed independently from errors (Baddeley et al., 2001; Emerson \& Miyake, 2003; but see Miyake, Emerson, Padilla, \& Ahn, 2004).

A particularly noteworthy aspect of the results of this experiment is the fact that the AS effect exhibited by the high sequence load group was similar for switch and noswitch trials. Thus, at least in this paradigm, AS does not seem to interfere with the process of switching between tasks per se. Rather, AS interferes with a function that is relevant on all trials alike. As we will argue, this function is the maintenance and/or updating of the appropriate task sequence.

One possible problem with our paradigm is that our primary tasks were relatively difficult (i.e., RTs $>1,000$ msec). Perhaps AS effects are absorbed in a subadditive manner into slack created by relatively long primarytask RTs. Thus, AS effects might be observed with easier tasks (as often are used in the task-switching literature), even in the absence of a sequencing load. In a follow-up experiment, we used a combination of color/ form discrimination tasks (Mayr, 2001) in an alternateruns paradigm with four spatial locations (i.e., low sequencing demand) and univalent versus bivalent stimulus blocks. We found no trace of an AS effect in either local or global costs, even though primary-task RTs were much smaller here (in the 700 -msec range). ${ }^{2}$ Thus, we feel confident that our basic result of a relatively small influence of AS on switching, as long as sequencing demands are low, generalizes to more typical task-switching situations.

Of additional interest is a direct comparison between Experiments 1 (or 2) and Experiment 3, because it illustrates how a contrast between no-switch and switch blocks confounds actual (local) switching demands with global processing demands. Mean RT for bivalent switching trials during AS in Experiment 3 was 1,692 msec (for the high sequencing load group), which is very similar to the mean RT for the corresponding condition in Experiment $1(1,708 \mathrm{msec})$. However, mean RTs for the equivalent no-switch times were very different $(1,435 \mathrm{msec}$ in Experiment 3 vs. 1,156 msec in Experiment 1). Presumably, this difference reflects demands involved with endogenous selection and maintenance of access to two different tasks, which is necessary in the no-switch condition in Experiment 3, but not in Experiment 1. The condition in Experiment 3 that is most comparable to the no-switch situation in Experiment 1 (i.e., not involving endogenous selection or sequencing) is the no-switch condition with univalent stimuli and low sequencing demands. Interestingly, the mean RT in this condition is $1,153 \mathrm{msec}$, which is very similar to that in the corre- sponding condition in Experiment $3(1,156 \mathrm{msec})$. Thus, as this informal comparison suggests, the combined sequence demand manipulation and the stimulus ambiguity manipulation within Experiment 3 were effective in bridging the gap between the blocked no-switch and switch conditions as used in Experiment 1 (and some of the former reports on AS effects on task switching).

\section{GENERAL DISCUSSION}

The results presented here are relevant for both the literature on task switching and the broader issue regarding the interplay between language and control. We will begin by looking at the implications of this work for models of task switching.

\section{Implications for Models of Task Switching}

The starting point for our study was the observation of very large effects of AS on switch costs reported by Baddeley et al. (2001) and Emerson and Miyake (2003). A straightforward conclusion from these results is that articulation, traditionally regarded as a nonexecutive slave system of working memory, plays a crucial role during executive task selection as assessed in the task-switching paradigm. The present results do not necessarily contradict this conclusion but suggest that it needs to be qualified in two ways.

First, the AS interference we obtained was less dramatic than that found in previous reports and was, in two of three experiments, confined to error effects. The question as to why, exactly, AS effects differed so much in magnitude across experiments needs to remain open here (see the Discussion section in Experiment 1). However, such a relatively small effect raises the question whether the relationship between language and control is as tight as suggested by the original reports.

Second, the present study provided much less evidence than previous reports have for AS effects on local switch costs (switch trials vs. no-switch trials). Furthermore, AS effects on global task selection costs were confined to conditions of high sequencing demand. If generalizable, these results seem to suggest that AS does not interfere with the process of changing task sets per se (see also Emerson \& Miyake, 2003). Of course, this conclusion is based on a null result, and there were some constellations in our data where AS effects on local switch costs might have been obtained with larger statistical power (e.g., the low sequence demand condition in Experiment 2).

Although we are not in a strong position to rule out the possibility that AS can interfere with the actual taskswitching process, our results do suggest that the actual switching process can proceed without the help of covert or overt articulation. In other words, there may be little need at this point to incorporate verbal-processing components as a necessary processing stage into models that deal with task switching (e.g., Gilbert \& Shallice, 2002; Meiran, 2001). 
There are two studies in the literature that seem inconsistent with this conclusion. Goschke (2000; see also Kray, Eber, \& Lindenberger, 2004) used a paradigm in which participants were cued on a trial-by-trial basis through symbolic cues to one of two tasks. In one condition, the participants were asked to verbalize the upcoming task label during the cue-stimulus interval, whereas in the other condition, they were required to verbalize a particular day of the week for each of the two cue symbols. Typically, participants can use a sufficiently long cue-stimulus interval to reduce their switch costs, usually referred to as a preparation effect. In this study, this preparation effect was obtained for the task label verbalization condition only, but not when the unrelated verbal response had to be produced. Goschke concluded from this result that verbal processing is regularly, and effectively, used during task preparation but is impaired when irrelevant verbal responses need to be produced. A potential problem with this interpretation is that using the cue to select an arbitrary word over and above the necessary task selection process imposes a dualselection demand that is not necessary when selecting the task-compatible task label. An important control condition would be one that keeps the selection demand constant but does not require a verbal response (e.g., a keypress response). In the absence of such a control condition, the result in Goschke is ambiguous.

Two additional relevant studies appeared after the initial version of this article had been completed. In a study by Miyake et al. (2004), a random-cuing paradigm in which participants switched between discriminating stimulus shapes and colors were used. Tasks were cued by either letters or task labels (color and shape). An increase of local costs was obtained for the AS condition when letters were used as task cues. The authors interpreted this result as evidence that verbalization is involved in retrieving the relevant task set, a process that should be more demanding with impoverished letter cues than with more direct task label cues (see also Mayr $\&$ Kliegl, 2000). Given the relevance of these results in the present context, we made an attempt to replicate them. We used the same tasks and dual-task conditions as Miyake et al., but we presented either word cues or letter cues in two separate between-subjects groups, both with short and long cue-stimulus intervals. We were unable to replicate the findings of Miyake et al., since neither of the two groups showed reliable AS effects on switch costs (as compared with foot tapping). ${ }^{3}$ Further research is needed to clarify whether verbalization subserves a retrieval function, a sequencing function, or both.

In the second relevant study, Saeki and Saito (2004b) used an alternate-runs paradigm in which participants switched between a numerical and a letter judgment task. Amount of external cuing was manipulated by presenting a line under the currently relevant stimulus in the cued condition. The results revealed AS effects that were highly similar for switch and no-switch trials. Consistent with our conclusions, the authors suggested that AS in- terferes with maintenance of a sequential plan. However, contrary to the present results, the AS effect was not reduced in the cued condition. A possible reason for this divergence in results is that the cue used in this study provided information about the currently relevant task, but not about the position in the sequence of tasks. Thus, combining our results and the findings from Saeki and Saito (2004b) provides at least indirect evidence that articulation subserves a specific sequencing function. It would certainly be of interest to directly compare cues that either do or do not provide sequential information.

\section{The Language-Control Relationship}

The second, broader issue touched upon by our results is the question of how language and control are related. Here, our results suggest some constraints on the range of possibilities opened up by earlier reports (Baddeley et al., 2001; Emerson \& Miyake, 2003). In our study, AS effects seemed nonspecific to switch trials and largely limited to situations in which high serial order demands were present. This suggests a relatively specific role for verbal processing during executive task managementnamely, the maintenance and/or updating of the relevant task sequence (see also Emerson \& Miyake, 2003).

Such a division of labor between task set selection and task sequencing makes sense from several theoretical perspectives. First, it is broadly consistent with the view introduced by Baddeley et al. (1984) that articulatory processing can serve as a working memory slave system that is at least partly independent of other slave systems and the so-called central executive. Second, the division between sequential control and task set selection is also consistent with the view that selection of task sets occurs through a constraint satisfaction process within a global workspace (see, e.g., Dehaene, Kerszberg, \& Changeux, 2001; Mayr, 2003). Purportedly, the result of this process is a single coherent task set representation, and all potentially competing task set representations are suppressed at this point. When taking this single task set constraint seriously, a problem arises as to whenever sequences of potentially competing tasks need to be performed. Obviously, the global workspace cannot represent a future task while filled up with a competing, presently relevant task. A possible solution could be a separate system that allows storage of action or task sequences, using a code that does not easily interfere with current task demands. An articulatory or phonological code not only allows for maintaining relatively neutral pointers to still-to-be-executed tasks, but due to its inherent sequential nature, also provides a natural repository for the representation of serial order.

Finally, the present results are of interest in the context of a recent study reported by Luria and Meiran (2003). These authors looked specifically at the process of maintaining and changing task order, using a psychological refractory paradigm in which task order was cued on a trial-by-trial basis. They found that changes in task order produced marked switch costs that were dependent on 
the cue-stimulus interval (i.e., a preparation effect), from which they concluded that task order was based on an "explicit representation" that could be activated in advance of stimulus presentation (thus, the preparation effect). Even though these authors did not mention language as a potential code for such a serial order plan, this idea seems highly compatible with their results. Future work that combines Luria and Meiran's paradigm with AS could, therefore, provide interesting corroborating information regarding the interplay between language, serial order control, and task set selection.

\section{Conclusion}

Vygotsky and Luria (1994) envisioned a broad role for language in organizing behavior and in making it independent from external stimuli. It is an important task for future work to determine the manner in which language does contribute to executive control of thought and action. The present work suggests that, at least in the adult cognitive system, the executive function of verbalization is rather specific. It seems to be linked to the sequential nature of the "secondary series of stimuli" language provides (Vygotsky \& Luria, 1994), allowing for control over the serial order of action rules.

\section{REFERENCES}

Baddeley, A. [D.], Chincotta, D., \& Adlam, A. (2001). Working memory and the control of action: Evidence from task switching. Journal of Experimental Psychology: General, 130, 641-657.

BADDELEY, A. D., LEWIS, V. J., \& VALLAR, G. (1984). Exploring the articulatory loop. Quarterly Journal of Experimental Psychology, 36A, 233-252.

Bates, T. C., \& D’Oliveiro, L. (2003). PsyScript: A Macintosh application for scripting experiments. Behavior Research Methods, Instruments, \& Computers, 35, 565-576.

Dehaene, S., Kerszberg, M., \& Changeux, J.-P. (2001). A neuronal model of a global workspace in effortful cognitive tasks. In P. C. Marijuán (Ed.), Cajal and consciousness: Scientific approaches to consciousness on the centennial of Ramón y Cajal's Textura (Annals of the New York Academy of Sciences, Vol. 929, pp. 152-165). New York: New York Academy of Sciences.

Emerson, M. J., \& MiYAKe, A. (2003). The role of inner speech in task switching: A dual-task investigation. Journal of Memory \& Language, 48, 148-168.

FAGOT, C. (1995). Chronometric investigations of task switching. Unpublished doctoral dissertation, University of California, San Diego.

Gehring, W. J., Bryck, R. L., Jonides, J., Albin, R. L., \& Badre, D. (2003). The mind's eye, looking inward? In search of executive control in internal attention shifting. Psychophysiology, 40, 572-585.

Gehring, W. J., Goss, B., Coles, M. G. H., Meyer, D. E., \& Donchin, E. (1993). A neural system for error detection and compensation. Psychological Science, 4, 385-390.

Gilbert, S. J., \& Shallice, T. (2002). Task-switching: A PDP model. Cognitive Psychology, 44, 297-337.

GoschKE, T. (2000). Intentional reconfiguration and involuntary persistence in task set switching. In S. Monsell \& J. Driver (Eds.), Control of cognitive processes: Attention and performance XVIII (pp. 331-356). Cambridge, MA: MIT Press.

JersiLD, A. T. (1927). Mental set and shift. Archives of Psychology, 14, $5-81$.

Kray, J., Eber, J., \& Lindenberger, U. (2004). Age differences in executive functioning across the lifespan: The role of verbalization in task preparation. Acta Psychologica, 115, 143-165.
Kray, J., \& Lindenberger, U. (2000). Adult age differences in task switching. Psychology \& Aging, 15, 126-147.

Luria, R., \& MeIRAN, N. (2003). Online order control in the psychological refractory period paradigm. Journal of Experimental Psychology: Human Perception \& Performance, 29, 556-574.

MAYR, U. (2001). Age differences in the selection of mental sets: The role of inhibition, stimulus ambiguity and response-set overlap. Psychology \& Aging, 16, 96-109.

MAYR, U. (2003). Towards principles of executive control: How mental sets are selected. In R. H. Kluwe, G. Lüer, \& F. Rösler (Eds.), Principles of learning and memory (pp. 223-240). Basel: Birkhäuser.

Mayr, U., \& Kliegl, R. (2000). Task-set switching and long-term memory retrieval. Journal of Experimental Psychology: Learning, Memory, \& Cognition, 26, 1124-1140.

MAYR, U., \& KLIEGL, R. (2003). Differential effects of cue changes and task changes on task-set selection costs. Journal of Experimental Psychology: Learning, Memory, \& Cognition, 29, 362-372.

MEIRAN, N. (1996). Reconfiguration of processing mode prior to task performance. Journal of Experimental Psychology: Learning, Memory, \& Cognition, 22, 1423-1442.

MEIRAN, N. (2001). Modeling cognitive control in task-switching. Psychological Research, 63, 234-249.

Miyake, A., Emerson, M. J., Padilla, F., \& Ahn, J. (2004). Inner speech as a retrieval aid for task goals: The effects of cue type and articulatory suppression in the random task cuing paradigm. Acta Psychologica, 115, 123-142.

RoGERS, R. D., \& MONSELL, S. (1995). Costs of a predictable switch between simple cognitive tasks. Journal of Experimental Psychology: General, 124, 207-231.

RUBinsteIn, J. S., MEYER, D. E., \& Evans, J. E. (2001). Executive control of cognitive processes in task switching. Journal of Experimental Psychology: Human Perception \& Performance, 27, 763-797.

SAEKI, E., \& SAITO, S. (2004a). Effect of articulatory suppression on task-switching performance: Implications for models of working memory. Memory, 12, 257-271.

SAEKI, E., \& SAITO, S. (2004b). The role of the phonological loop in task-switching performance: The effect of articulatory suppression in the alternating runs paradigm. Psychologia, 47, 35-43.

SPECTOR, A., \& Biederman, I. (1976). Mental set and mental shift revisited. American Journal of Psychology, 89, 669-679.

VygotSKY, L. S., \& LURIA, A. R. (1994). Tool and symbol in child development. In R. van der Veer \& J. Valsiner (Eds.), The Vygotsky reader (pp. 99-174). Oxford: Blackwell.

Zbrodoff, N. J., \& LoGAN, G. D. (1986). On the autonomy of mental processes: A case study of arithmetic. Journal of Experimental Psychology: General, 115, 118-130.

\section{NOTES}

1. Initially, an RSI of $50 \mathrm{msec}$ was intended for all the participants. However, a programming error was discovered after running the first 12 participants in Experiment 1, in which the RSI had unintentionally been set to $250 \mathrm{msec}$. Thus, the 50- and 250-msec RSIs were split equally across participants, conditions, and counterbalancing orders. Later analyses showed no significant differences between the two RSI groups in any of the three experiments. Thus, the two groups were collapsed in all the subsequent analyses and descriptions of results.

2. In this experiment, essentially the same procedure as that in Experiment 3 was used, except only a low sequencing load was used and the tasks used were form discrimination (horizontal and vertical alignment) versus color discrimination (blue and red); 24 participants were tested. Neither the general effect of AS (AS contrast $\times$ stimulus ambiguity interaction) nor the interaction with switching (AS contrast $\times$ stimulus ambiguity $\times$ switching) produced reliable interactions for the RT or the error measure (all $F \mathrm{~s}<1.0$ ).

3. Twelve subjects each worked on eight blocks of 56 trials (after practice) with either task label word cues or letter cues on the color and the shape task as in Miyake et al. (2004). Cue-stimulus interval was manipulated in a blocked manner (200 msec vs. 1,200 msec). In the long 
cue-stimulus interval condition, which was comparable to the one used by Miyake et al. (2004), switch costs were $31 \mathrm{msec}$ ( $2.0 \%$ error costs) for foot tapping and $32 \mathrm{msec}(1.0 \%$ error costs) for AS in the word cue condition and $128 \mathrm{msec}(3.6 \%$ error costs) and $116 \mathrm{msec}(2.3 \%$ error costs) in the letter cue condition, respectively. In the short cue-stimulus interval condition, switch costs were $72 \mathrm{msec}(2.1 \%$ error costs) for foot tapping and $36 \mathrm{msec}(2.8 \%$ error costs) for AS in the word cue condition and $145 \mathrm{msec}(2.1 \%$ error costs $)$ and $199 \mathrm{msec}(2.8 \%$ error costs $)$ in the letter cue condition, respectively. The AS-related increase in RT switch costs in the letter condition was far from reliable (RT, $p>.5$; er- rors, $p>$.6); however, given relatively low power, we cannot make strong claims about the absence of effects here. Thus, it is possible that in the short-interval condition (where retrieval demands may be particularly high), an AS effect would become apparent with a larger sample size. However, in the long cue-stimulus interval condition we found no trace of an AS effect that was comparable to the situation reported in Miyake et al. (2004).

(Manuscript received August 31, 2003; revision accepted for publication August 6, 2004.) 